\title{
Rapid Akt activation by nicotine and a tobacco carcinogen modulates the phenotype of normal human airway epithelial cells
}

\author{
Kip A. West, ${ }^{1}$ John Brognard, ${ }^{1}$ Amy S. Clark, ${ }^{1}$ Ilona R. Linnoila, ${ }^{2}$ Xiaowei Yang, ${ }^{1}$ \\ Sandra M. Swain, ${ }^{1}$ Curtis Harris, ${ }^{3}$ Steven Belinsky, ${ }^{4}$ and Phillip A. Dennis ${ }^{1}$ \\ ${ }^{1}$ Cancer Therapeutics Branch, \\ ${ }^{2}$ Cell and Cancer Biology Branch, and \\ ${ }^{3}$ Laboratory of Human Carcinogenesis, Center for Cancer Research, National Cancer Institute, Bethesda, Maryland, USA \\ ${ }^{4}$ Lovelace Respiratory Research Institute, Albuquerque, New Mexico, USA
}

Tobacco-related diseases such as lung cancer cause over 4.2 million deaths annually, with approximately 400,000 deaths per year occurring in the US. Genotoxic effects of tobacco components have been described, but effects on signaling pathways in normal cells have not been described. Here, we show activation of the serine/threonine kinase Akt in nonimmortalized human airway epithelial cells in vitro by two components of cigarette smoke, nicotine and the tobacco-specific carcinogen 4-(methylnitrosamino)-1-(3-pyridyl)-1-butanone (NNK). Activation of Akt by nicotine or NNK occurred within minutes at concentrations achievable by smokers and depended upon $\alpha_{3}-/ \alpha_{4}$-containing or $\alpha_{7}$-containing nicotinic acetylcholine receptors, respectively. Activated Akt increased phosphorylation of downstream substrates such as GSK-3, p70 ${ }^{\text {S6K }}$, 4EBP-1, and FKHR. Treatment with nicotine or NNK attenuated apoptosis caused by etoposide, ultraviolet irradiation, or hydrogen peroxide and partially induced a transformed phenotype manifest as loss of contact inhibition and loss of dependence on exogenous growth factors or adherence to ECM. In vivo, active Akt was detected in airway epithelial cells and lung tumors from NNK-treated A/J mice, and in human lung cancers derived from smokers. Redundant Akt activation by nicotine and NNK could contribute to tobacco-related carcinogenesis by regulating two processes critical for tumorigenesis, cell growth and apoptosis.

J. Clin. Invest. 111:81-90 (2003). doi:10.1172/JCI200316147.

\section{Introduction}

The serine/threonine kinase Akt (or protein kinase B), controls key cellular processes such as glucose metabolism, cell cycle progression, and apoptosis (1), and active Akt can contribute to tumorigenesis in vivo in lymphoid, breast, ovarian, prostate, and brain tissues (2). Although gene amplification or mutations in Akt are infrequent in human cancers, Akt is commonly activated in tumor cells through activation of growth factor receptors or Ras, or inactivation of phosphatase-and-

Received for publication June 11, 2002, and accepted in revised form October 22, 2002.

Address correspondence to: Phillip A. Dennis, National Cancer Institute/Naval Medical Oncology, Building 8, Room 5101, 8901 Wisconsin Avenue, Bethesda, Maryland 20889, USA. Phone: (301) 496-0901; Fax: (301) 496-0047;

E-mail: pdennis@nih.gov.

Kip A. West and John Brognard contributed equally to this work.

Conflict of interest: The authors have declared that no conflict of interest exists.

Nonstandard abbreviations used: phosphatase-and-tensinhomologue-deleted-on-chromosome-10 (PTEN); non-small cell lung cancer (NSCLC); 4-(methylnitrosamino)-1-(3-pyridyl)-1butanone (NNK); normal human bronchial epithelial cell (NHBE); small airway epithelial cell (SAEC); nicotinic acetylcholine receptor (nAchR); dihydro- $\beta$-erythroidine $(\mathrm{DH} \beta \mathrm{E})$;

$\alpha$-bungarotoxin ( $\alpha$-BTX); methyllycaconitine (MLA); mecamylamine (MCA); $\alpha$-anatoxin $(\alpha$-ATX); glycogen synthase kinase-3 (GSK-3). tensin-homologue-deleted-on-chromosome-10 (PTEN), a phosphatase whose tumor suppressor function depends on dephosphorylation of $3^{\prime}$-phosphoinositide products of the upstream kinase PI3K (3). Full activation of Akt requires phosphorylation at two sites, one in the activation domain (T308) and one in the C-terminal hydrophobic motif (S473). To date, Akt activation has not been associated with environmental carcinogenesis. Our laboratory recently identified constitutive activation of the PI3K/Akt pathway in $90 \%$ of non-small cell lung cancer (NSCLC) cell lines and showed that Akt activity promoted cellular survival and resistance to chemotherapy or $\gamma$-irradiation (4). Because lung cancer is an environmental cancer commonly associated with tobacco use, we hypothesized that Akt activation might be associated with tobacco-related carcinogenesis and might be related to exposure to tobacco components.

Traditional models of tobacco-related tumorigenesis are genocentric, in that tobacco components promote carcinogenesis through a multistep process that involves exposure to and activation of carcinogens such as 4-(methylnitrosamino)-1-(3-pyridyl)-1-butanone (NNK) or polyaromatic hydrocarbons, which leads to formation of DNA adducts. If DNA repair is intact, normal DNA structure can be restored, but if adducts persist, cells with damaged DNA will initiate a process of self-destruction through apoptosis. Executing that 
decision to undergo apoptosis will protect the organism from further carcinogenic events occurring in the damaged cell. In contrast, if adducts persist without the execution of apoptosis, mutations in key genes such as $p 53, p 16$, or $K$-ras can accumulate, culminating in tumor formation. Therefore, identifying mechanisms that promote cellular survival in the setting of DNA damage might elucidate critical steps in carcinogenesis.

In the studies described within, we demonstrate an early biochemical effect of tobacco components in normal human lung epithelial cells: activation of the PI3K/Akt pathway. To test activation of Akt in an in vitro model system, we established two sets of primary human airway epithelial cell cultures: normal human bronchial epithelial cells (NHBEs) derived from large airways, which are the precursor cells for squamous cell carcinomas; and small airway epithelial cells (SAECs), which are the precursor cells for adenocarcinomas. In each cell type, two components of tobacco were tested: nicotine, the addictive component of tobacco and the precursor to many tobacco carcinogens; and NNK, a potent, tobacco-specific carcinogen. Nicotine or NNK activated Akt in NHBEs and SAECs at nanomolar doses within minutes. Multiple $\alpha$ and $\beta$ subunits of nicotinic acetylcholine receptors (nAchRs), which bind nicotine and NNK, were expressed in NHBEs and SAECs. Using pharmacologic inhibitors, we showed that nicotinic activation of Akt depends upon PI3K and specific nAchRs. Once activated by tobacco components, Akt increased phosphorylation of downstream substrates, increased cellular survival, and conferred some properties of transformed cells. We extended these studies by showing activation of Akt in vivo in NNK-treated $\mathrm{A} / \mathrm{J}$ mice and in human lung cancers from smokers. Our studies are consistent with the hypothesis that carcinogens promote tumorigenesis through both biochemical and genetic mechanisms.

\section{Methods}

Cell culture. Nonimmortalized NHBEs and SAECs (Cambrex Bioscience, Walkersville, Maryland, USA) were maintained in culture with growth supplements (unless otherwise indicated) according to the manufacturer's recommendations. Lot numbers used for NHBE studies were 1131, 2095, and 1452. The lot number for the SAECs was 0894. All lots were derived from nonsmokers. Pharmacologic treatments. For time-dependent induction of Akt phosphorylation, $100 \mu \mathrm{M}$ nicotine (SigmaAldrich, St. Louis, Missouri, USA) or $1 \mu \mathrm{M}$ NNK (ChemSyn Laboratories, Lenexa, Kansas, USA) was used. Dose-response curves were generated at $60 \mathrm{~min}$ utes for NHBEs and 45 minutes for SAECs. To assess phosphorylation of downstream substrates, $10 \mu \mathrm{M}$ nicotine was added for 60 minutes or $1 \mu \mathrm{M}$ NNK was added for 45 minutes. For competition in kinase assays, $10 \mu \mathrm{M} \mathrm{LY} 294002$ (Sigma-Aldrich), $10 \mu \mathrm{M}$ dihydro- $\beta$-erythrodine (DH $\beta$ E; Sigma-Aldrich), or $100 \mathrm{nM}$ $\alpha$-bungarotoxin ( $\alpha$-BTX; Sigma-Aldrich) was added 30 minutes before nicotine or NNK. To assess the role of different nAchRs in mediating Akt activity, $10 \mu \mathrm{M}$ nicotine was added for 60 minutes with or without 30 minutes' pretreatment with $10 \mu \mathrm{M}$ LY294002, $1 \mu \mathrm{M}$ $\alpha$-BTX, $1 \mu \mathrm{M}$ methylallylaconitine (MLA; SigmaAldrich), $50 \mu \mathrm{M}$ mecamylamine (MCA; SigmaAldrich), or $10 \mu \mathrm{M}$ DH $\beta$ E. To confirm the role of $\alpha_{3}$ nAchRs in activating Akt, $10 \mu \mathrm{M} \alpha$-anatoxin ( $\alpha$-ATX; Sigma-Aldrich) was added to NHBEs for 30 minutes with or without 30 minutes' pretreatment with $10 \mu \mathrm{M}$ DH $\beta$ E. Inhibition studies were also performed with 1 $\mu \mathrm{M}$ NNK (45 minutes), except that lower doses of nAchR antagonists were used (100 nM $\alpha$-BTX, $100 \mathrm{nM}$ MLA, $10 \mu \mathrm{M}$ MCA). For examination of the effects on apoptosis, NHBEs were pretreated for 45 minutes with $10 \mu \mathrm{M}$ LY294002, and/or treated for 45 minutes with $10 \mu \mathrm{M}$ nicotine, and/or treated for 24 hours with 20 $\mu \mathrm{M}$ etoposide (Calbiochem-Novabiochem Corp., La Jolla, California, USA). Cells were harvested for flow cytometry as described previously (4).

Immunoblotting. After the various pharmacologic treatments described above, cell extracts were prepared as described previously (4). Protein yield was quantified using the Bio-Rad DC protein assay kit (Bio-Rad Laboratories Inc., Hercules, California, USA). Equivalent protein was loaded, and the lysates were separated by SDS-PAGE and transferred to nitrocellulose membranes. Equivalent loading was confirmed by staining of membranes with fast green. Membranes were blocked for 1 hour in blocking buffer $(1 \times$ Tris buffered saline [TBS], $5 \%$ milk, $0.20 \%$ Tween- 20$)$ and placed in primary antibody ( $1 \times \mathrm{TBS}, 5 \%$ milk, $0.10 \%$ Tween- 20 , 1:1,000 antibody) overnight at $4{ }^{\circ} \mathrm{C}$. Membranes were washed three times in wash buffer $(0.10 \%$ NP40, $0.10 \%$ Tween-20, 1× TBS). Primary antibody was detected using horseradish peroxidase-linked goat anti-mouse or goat anti-rabbit IgG antibodies and visualized with the enhanced chemiluminescent detection system ECL (Amersham Pharmacia Biotech, Amersham, United Kingdom). All phosphospecific antibodies were from Cell Signaling Technology Inc. (Beverly, Massachusetts, USA) and were used at 1:1,000 dilutions. Immunoblot experiments were performed at least three times.

Akt kinase assays. In vitro kinase assays using Akt kinase assay kits (Cell Signaling Technology Inc.) were performed as described previously (4) after $10 \mu \mathrm{M}$ nicotine or $1 \mu \mathrm{M}$ NNK administration. Ten micromolar LY294002, $10 \mu \mathrm{M}$ DHßE, or $100 \mathrm{nM} \alpha$-BTX was added 45 minutes before nicotine or NNK, and samples were harvested 45 minutes later.

$R T-P C R$. Total-RNA extraction and PCR reaction mixtures were as described previously (4). Subunit-specific primers for nAchRs were synthesized by Sigma-Genosys (The Woodlands, Texas, USA) with the following sequences: $\alpha_{1}: 5^{\prime}$-CGTCTGGTGGCAAAGCT- $3^{\prime}$ (sense), $5^{\prime}$ CCGCTCTCCATGAAGTT-3' (antisense); $\alpha_{2}$ : $5^{\prime}$-CCGGTGGCTTCTGATGA-3' (sense), $5^{\prime}$-CAGATCATTCCAGCTAGG-3' (antisense) (5); $\alpha_{3}: 5^{\prime}$-CCATGTCTCAGCTGGTG-3' (sense), $5^{\prime}$-GTCCTTGAGGTTCATGGA-3' (antisense) (6); $\alpha_{4}: 5^{\prime}$ CTCTCGAACACCCACTC-3' (sense), 5'-AGCAGGCTCCCG- 
GTCCCT-3' (antisense) (7); $\alpha_{5}: \quad 5^{\prime}$-TCATGTAGACAGGTACTTC-3' (sense), $5^{\prime}$-ATTTGCCCATTTATAAATAA-3' (antisense) (8); $\alpha_{6}: 5^{\prime}$-GGCCTCTGGACAAGACAA- $3^{\prime}$ (sense), $5^{\prime}$-AAGATTTTCCTGTGTTCCC-3' (antisense) (5); $\alpha_{7}: 5^{\prime}$ CACAGTGGCCCTGCAGACCGATGGTACGGA-3' (sense), $5^{\prime}$ CTCAGTGGCCCTGCTGACCGATGGTACGGA-3' (antisense) (9); $\alpha_{9}$ : 5'-GTCCAGGGTCTTGTTTGT- $3^{\prime}$ (sense), $5^{\prime}$-ATCCGCTCTTGCTATGAT- $3^{\prime}$ (antisense) (6); $\alpha_{10}$ : $5^{\prime}$-CTGTTCCGTGACCTCTTT-3' (sense), $5^{\prime}$-GGAAGGCTGCTACATCCA$3^{\prime}$ (antisense) (6); $\beta_{2}: 5^{\prime}$-CAGCTCATCAGTGTGCA-3' (sense), $5^{\prime}$-GTGCGGTCGTAGGTCCA-3' (antisense) (5); $\beta_{3}: 5^{\prime}$ AGAGGCTCTTTCTGCAGA-3' (sense), $5^{\prime}$-GCCACATCTTCAAAGCAG-3' (antisense) (5); $\beta_{4}: 5^{\prime}$-CTGAAACAGGAATGGACT- $3^{\prime} \quad$ (sense), $5^{\prime}$-CCATGTCTATCTCCGTGT- $3^{\prime}$ (antisense) (5); and $\beta$-actin primers were $5^{\prime}$-GTGGGGCGCCCCAGGCACCA-3' (sense) and 5'-CTCCTTAAGTCACGCACGATTTC-3' (antisense) (Sigma-Genosys). L. Lustig (Johns Hopkins University, Baltimore, Maryland, USA) generously provided $\alpha_{9}$ and $\alpha_{10}$ cDNA controls. nAchR primers generated predicted products of $505 \mathrm{bp}$ $\left(\alpha_{1}\right), 466$ bp $\left(\alpha_{2}\right), 401$ bp $\left(\alpha_{3}\right), 371$ bp $\left(\alpha_{4}\right), 265$ bp $\left(\alpha_{5}\right), 413$ bp $\left(\alpha_{6}\right), 598$ bp $\left(\alpha_{7}\right), 403 \mathrm{bp}\left(\alpha_{9}\right), 388 \mathrm{bp}\left(\alpha_{10}\right), 347 \mathrm{bp}\left(\beta_{2}\right)$, $354 \mathrm{bp}\left(\beta_{3}\right)$, and $310 \mathrm{bp}\left(\beta_{4}\right)$. No differences in PCR products were observed in different lots of NHBEs.

Anoikis assays. Anoikis assays were performed using the method of Bretland et al. with modifications (10). Briefly, 96-well tissue culture plates were coated with poly-HEME [poly-(2-hydroxyethylmethacrylate); SigmaAldrich]. NHBEs were plated on poly-HEME-coated plates in DMEM with $0.1 \%$ BSA with or without $10 \mu \mathrm{M}$ nicotine and/or pretreatment with $10 \mu \mathrm{M}$ LY294002 or $10 \mu \mathrm{M}$ DH $\beta E$ for 45 minutes. After 8 hours, cells were harvested for assessment of anoikis using CellDeath ELISA kits (Roche Diagnostics Corp., Indianapolis, Indiana, USA) according to the manufacturer's instructions. Similar incubations were performed with $1 \mu \mathrm{M}$ NNK with substitution of $100 \mathrm{nM} \alpha$-BTX for DH $\beta$ E.

Ultraviolet irradiation assays. NHBEs were plated in triplicate onto 96-well plates in complete media and allowed to attach overnight. The following day, cells were washed, and complete media or serum-free DMEM media containing 0.1\% BSA were added. Cells were pretreated, or not, with $10 \mu \mathrm{M}$ LY294002 or 10 $\mu \mathrm{M} \mathrm{DH} \beta \mathrm{E}$ for 45 minutes, and treated with or without $10 \mu \mathrm{M}$ nicotine for 45 minutes. Cells were irradiated (4 $\mathrm{kJ} / \mathrm{m}^{2} \mathrm{UV}$ radiation) with a Stratalinker UV Crosslinker (Stratagene, La Jolla, California, USA) containing UV light bulb sources $5 \times 254 \mathrm{~nm}$ in area. Twenty-four hours after irradiation, apoptosis was measured using CellDeath ELISA kits.

Tumor specimens. For immunohistochemical analysis of Akt phosphorylation, A/J mice were given PBS vehicle or $9.1 \mathrm{mg}$ of the tobacco-specific nitrosamine NNK in drinking water for 8 weeks and then serially sacrificed (11). Lung tissues were fixed in $10 \%$ neutral formalin and embedded in paraffin, and 5-mm sections were stained for phospho-Akt immunoreactivity. For immunoblotting analysis, frozen specimens from lungs of $\mathrm{A} / \mathrm{J}$ mice treated with intraperitoneal injections of
PBS or NNK (12) were used. Mouse studies were approved by the NIH Animal Care and Use Committee. Human lung cancer specimens from smokers were obtained after institutional review board approval of protocol no. 0298229 from the University of Maryland (College Park, Maryland, USA) under a contractual agreement between the National Cancer Institute and the University of Maryland. Paraffin-embedded sections of these lung cancer specimens were stained for phospho-S473 immunoreactivity.

Immunohistochemistry. The immunohistochemical methods for formalin-fixed and paraffin-embedded sections have been described previously (13). For phosphospecific antibodies, antigen retrieval was performed in $10 \mathrm{mM}$ Tris- $\mathrm{HCl}$ buffer, $\mathrm{pH} 8.0$, for 20 minutes in $95^{\circ} \mathrm{C}$ water bath. After antigen retrieval, the tissue sections were incubated with phospho-Akt (S473) antibodies at 1:50 dilution overnight at $4^{\circ} \mathrm{C}$. The binding of antibodies to their antigenic sites in the tissue sections was amplified with the use of biotinylated goat anti-rabbit antibodies and avidin-peroxidase conjugate for 30 minutes (VECTASTAIN Elite ABC kits; Vector Laboratories Inc., Burlingame, California, USA), followed by reaction with 3,3'-diaminobenzidine (SigmaAldrich). Tissue sections were counterstained with Mayer's hematoxylin (BioGenex Laboratories, San Ramon, California, USA).

\section{Results}

Nicotine and NNK induce Akt phosphorylation. To investigate whether components of tobacco could activate Akt, we chose to test nicotine and NNK, a nicotinederived, tobacco-specific nitrosamine with high carcinogenic potential (14), from over 3,500 compounds in the particulate fraction of tobacco smoke. When added to NHBEs or SAECs in vitro, nicotine increased Akt phosphorylation at S473 and T308 in a time- and dose-dependent manner (Figure 1, a and b). Although stimulation of Akt phosphorylation by nicotine in each cell type was evident within 5 minutes, the patterns of S473 and T308 phosphorylation were different over time (Figure 1a, upper panels). Nicotine maximally increased $S 473$ phosphorylation at 60 minutes in NHBEs and 30 minutes in SAECs, and increased S473 phosphorylation was maintained for 24 hours (see Figure 4a, inset). Phosphorylation of T308 by nicotine was more transient, reaching a maximum at 30 minutes in NHBEs and 15 minutes in SAECs, with decreases observed at subsequent time points. In experiments designed to test dose-dependent responses to nicotine, nicotine increased Akt phosphorylation in both cell types with doses as low as 10-100 nM, but maximum phosphorylation was observed at $1-10 \mu \mathrm{M}$ (Figure 1b). These concentrations are achievable in smokers, since average steady-state serum concentrations of nicotine have been reported at $200 \mathrm{nM}$, and acute increases to $10-100 \mu \mathrm{M}$ in serum or to $1 \mathrm{mM}$ at the mucosal surface immediately after smoking have been reported (15-17). 
a

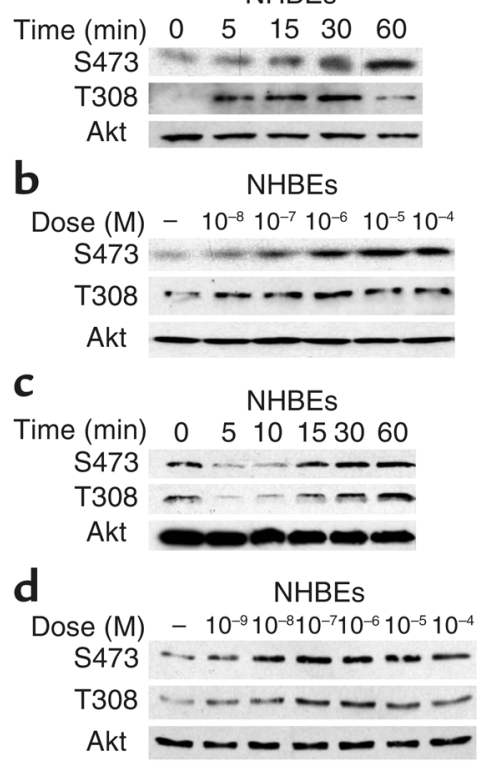

SAECs

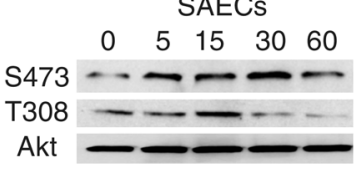

SAECS

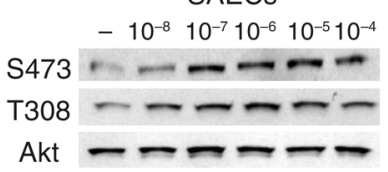

SAECS

$0 \quad 5 \quad 10153060$

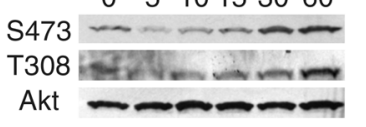

SAECS

- $10^{-9} 10^{-8} 10^{-7} 10^{-6} 10^{-5} 10^{-4}$

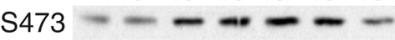

T308 - - - - - - .

\section{Figure 1}

Phosphorylation of Akt by nicotine or NNK in NHBEs and SAECs. (a) Nicotine increased Akt phosphorylation in a timedependent manner in NHBEs (left panels) and SAECs (right panels), as assessed by immunoblotting with anti-phosphoS473, anti-phospho-T308, and anti-Akt antibodies. (b) Nicotine-induced Akt phosphorylation was also dosedependent. (c) NNK increased Akt phosphorylation in a time-dependent manner. (d) NNK-induced Akt phosphorylation was also dose-dependent.
The tobacco-specific carcinogen NNK stimulated Akt phosphorylation with different kinetics and was more potent (Figure 1, $\mathrm{c}$ and d). The time course of NNKinduced Akt phosphorylation was similar for NHBEs and SAECs and for both sites of phosphorylation (Figure 1c). For the first 10-15 minutes, we observed decreased S473 and T308 phosphorylation after NNK administration, followed by increased S473 and T308 phosphorylation that was maximal at 60 minutes. Unlike nicotine induction of S473 phosphorylation, which was maintained for at least 24 hours, NNKinduced S473 phosphorylation was maintained for 8 hours (data not shown). NNK dose-dependence was also similar for NHBEs and SAECs. Increased phosphorylation of both sites was observed with doses as low as $1 \mathrm{nM}$ (Figure 1d), with maximal induction at $100 \mathrm{nM}$ for NHBEs and $10 \mathrm{nM}$ for SAECs. Although these data showed that nicotine or NNK could induce Akt activation in NHBEs and SAECs, the differences in dose- and time-dependence of nicotine- and NNKinduced Akt phosphorylation raised the possibility that that these compounds might use different mechanisms to activate Akt.

Effects of nicotine or NNK on Akt kinase activity and phosphorylation of downstream substrates. To confirm that increased Akt phosphorylation at S473 and T308 was indicative of increased kinase activity, we measured the phosphorylation of an exogenous peptide substrate, glycogen synthase kinase- $3 \alpha / \beta($ GSK- $3 \alpha / \beta)$, after nicotine or NNK administration (Figure 2a). Nicotine or NNK increased Akt kinase activity in a PI3K-dependent manner, since the PI3K inhibitor LY294002 (18) inhibited induction of Akt activity by nicotine or NNK. To demonstrate that Akt activation propagated signaling cascades within NHBEs, and to identify substrates or cellular processes that might be affected by nicotine- or NNK-mediated Akt activation, we assessed phosphory- lation of five proteins previously identified to be downstream of Akt at the time of maximal nicotinic Akt induction: a member of the forkhead transcription factor family (FKHR), GSK- $3 \alpha$ and $-3 \beta$, a ribosomal kinase $\left(\mathrm{p} 70^{\mathrm{S} 6 \mathrm{~K}}\right)$, and a binding protein for eukaryotic translation initiation factor 4E (4EBP-1) (Figure 2b). We observed different patterns of phosphorylation induced by nicotine or NNK. Nicotine increased the phosphorylation of all downstream substrates, but phosphorylation of GSK-3 $\alpha$ and FKHR was induced most. NNK increased phosphorylation of all downstream substrates except FKHR and had the greatest effects on phospho-
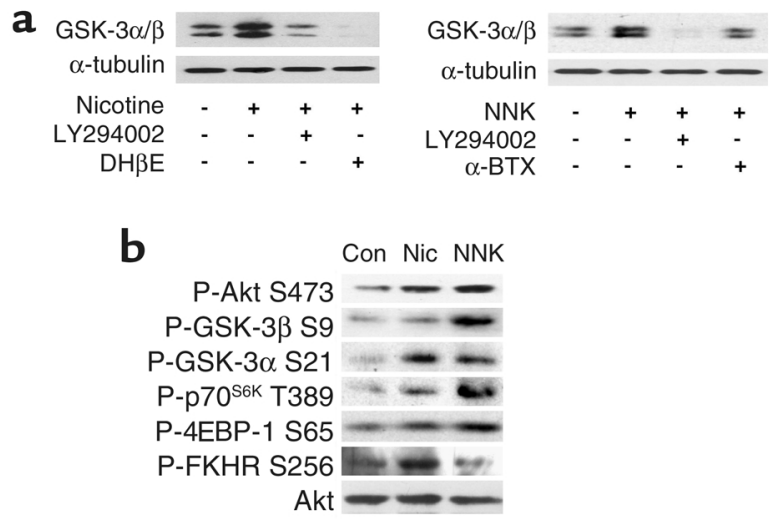

Figure 2

Akt kinase activity and effect on downstream substrates. (a) We measured Akt kinase activity in NHBEs by immunoprecipitating active Akt and assessing phosphorylation of an exogenous peptide, GSK- $3 \alpha / \beta$, after administration of nicotine (left panels) or NNK (right panels). LY294002, DH $\beta E$ (an $\alpha_{3} / \alpha_{4}$ nAchR antagonist), or $\alpha$-BTX (an $\alpha_{7}$ $n A c h R$ antagonist) inhibited nicotinic induction of Akt kinase activity. (b) Phosphorylation of substrates downstream of Akt in NHBEs was increased after administration of nicotine (Nic; middle lane) or NNK (right lane), compared with that in untreated cells (Con), as assessed by immunoblotting with the indicated phosphospecific antibodies. 
Table 1

RT-PCR expression of $n A c h R$ subunits

\begin{tabular}{lccc}
\cline { 2 - 2 } & SAEC & NHBE & H157 \\
$\alpha_{1}$ & - & - & - \\
$\alpha_{2}$ & + & - & - \\
$\alpha_{3}$ & - & + & + \\
$\alpha_{4}$ & + & - & + \\
$\alpha_{5}$ & - & + & + \\
$\alpha_{6}$ & - & - & + \\
$\alpha_{7}$ & + & + & + \\
$\alpha_{9}$ & + & + & + \\
$\alpha_{10}$ & + & + & - \\
$\beta_{2}$ & + & + & + \\
$\beta_{3}$ & - & - & - \\
$\beta_{4}$ & + & + & - \\
\hline
\end{tabular}

rylation of GSK-3 3 and $p 70^{\mathrm{S} 6 \mathrm{~K}}$. The different induction of phosphorylation of downstream substrates further suggested that the kinetics and/or the mechanism of activation of Akt for nicotine and NNK are different. Together with other studies that investigated the effects of Akt activation on these substrates (19-21), our results support the hypothesis that activation of Akt by nicotine or NNK promotes cell cycle progression, increases protein synthesis, and inhibits apoptosis.

Expression of $n A c b R$ subunits in NHBEs and SAECs. Because nAchRs bind nicotine and NNK and mediate the biologic effects of these tobacco components, we characterized expression of nAchR subunits in NHBEs and SAECs. nAchRs belong to the superfamily of ligandgated ion channels that are predominantly expressed in neural tissue, but they have recently been reported to be expressed in other tissues $(22,23)$. Functional nAchRs are composed of homopentamers derived from subunits $\alpha_{7}-\alpha_{10}$ or heteropentamers derived from six $\alpha$ subunits $\left(\alpha_{1}-\alpha_{6}\right)$ and three $\beta$ subunits $\left(\beta_{2}-\beta_{4}\right)$. nAchRs containing $\alpha_{3}$ or $\alpha_{4}$ are most abundant in neural tissue (24), and $\alpha_{7^{-}}$ containing nAchRs have been described in human bronchial epithelial and endothelial cells (6). We performed nAchR subunit-specific RT-PCR analysis of sub- units $\alpha_{1}-\alpha_{10}$ and $\beta_{2}-\beta_{4}$ in SAECs, NHBEs, and an NSCLC cell line (H157). Cell type-specific nAchR subunit expression was observed and is summarized in Table 1 . SAECs selectively express $\alpha_{2}$ and $\alpha_{4}$ subunits, and NHBEs selectively express $\alpha_{3}$ and $\alpha_{5}$ subunits. Both cell types express $\alpha_{7}-\alpha_{10}, \beta_{2}$, and $\beta_{4}$ subunits. These results imply that SAECs and NHBEs express similar homopentamer $n$ AchRs containing $\alpha_{7}-\alpha_{10}$ subunits but likely express a different repertoire of heteropentamers: namely $\alpha_{3} \beta_{4}, \alpha_{3} \beta_{4} \alpha_{5}$, or $\alpha_{3} \beta_{2}$ nAchRs in NHBEs, and $\alpha_{2} \beta_{2}, \alpha_{2} \beta_{4}, \alpha_{4} \beta_{2}$, or $\alpha_{4} \beta_{4}$ nAchRs in SAECs.

Effects of nicotinic antagonists on Akt phosphorylation. To determine which nAchRs might facilitate nicotinic activation of Akt, we treated NHBEs and SAECs with pharmacologic inhibitors directed against specific $\alpha$ subunit-containing nAchRs and measured Akt activation after treatment with nicotine or NNK. (LY294002 served as a positive control in these studies and inhibited both basal and nicotine-induced S473 phosphorylation.) In NHBEs and SAECs (Figure 3a, upper left and lower panels), the $\alpha_{7}$ antagonist $\alpha$-BTX (25), the $\alpha_{7}$ antagonist MLA, or the nonspecific nAchR antagonist MCA did not attenuate nicotine-induced Akt phosphorylation, either at lower doses where specificity is greatest (data not shown) or at the higher doses shown. In contrast, the $\alpha_{3} / \alpha_{4}$ antagonist $\operatorname{DH} \beta E(26,27)$ inhibited nicotine-induced Akt phosphorylation (Figure 3a) and activity (Figure 2a, left panels), suggesting that nAchRs containing $\alpha_{3}$ or $\alpha_{4}$ subunits (in NHBEs or SAECs, respectively) mediate Akt induction by nicotine. Increased Akt phosphorylation was occasionally observed with addition of nicotinic antagonists alone, and this may be related to the observation that nicotinic antagonists such DH $B$ E and MLA can cause a compensatory increase in expression and activation of other, nontargeted nAchRs (28). The role of $\alpha_{3}$-containing nAchRs in nicotine-induced Akt phosphorylation in NHBEs was further supported by the observation that a specific $\alpha_{3}$ nicotinic agonist, $\alpha$-ATX (29), increased Akt phosphorylation in NHBEs, and that this effect was inhibited by DHßE (Figure 3a, upper right panels).

\section{Figure 3}

Effect of $n A$ chR antagonists on nicotinic activation of Akt in NHBEs and SAECs. (a) Nicotine. Only LY294002 or the $\alpha_{3} / \alpha_{4}$ antagonist $\mathrm{DH} \beta \mathrm{E}$ inhibited nicotine-induced Akt phosphorylation in NHBEs (upper left panels) and SAECs (lower panels). To confirm the role of $\alpha_{3}$ nAchRs in activating Akt in NHBEs, $\alpha$-ATX (an $\alpha_{3}$ agonist) was added to NHBEs with or without $\mathrm{DH} \beta \mathrm{E}$ (upper right panels). (b) NNK. In contrast to nicotine-mediated Akt phosphorylation, NNK-induced phosphorylation in NHBEs was inhibited by LY294002, the $\alpha_{7}$ antagonists $\alpha$-BTX and MLA, and the nonspecific inhibitor MCA. DH $\beta$ E was ineffective.

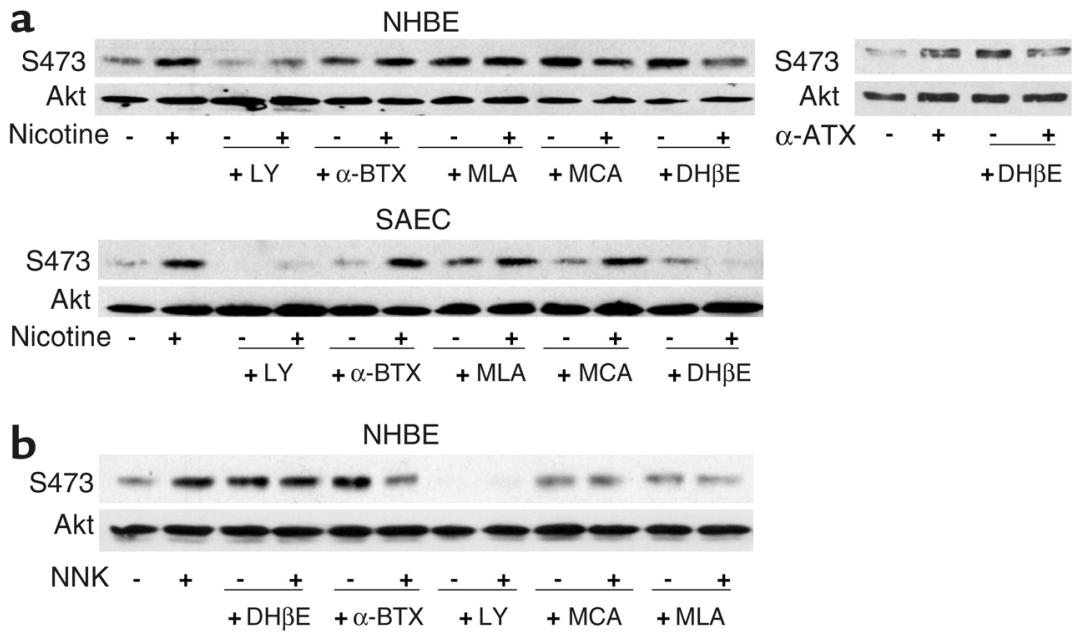



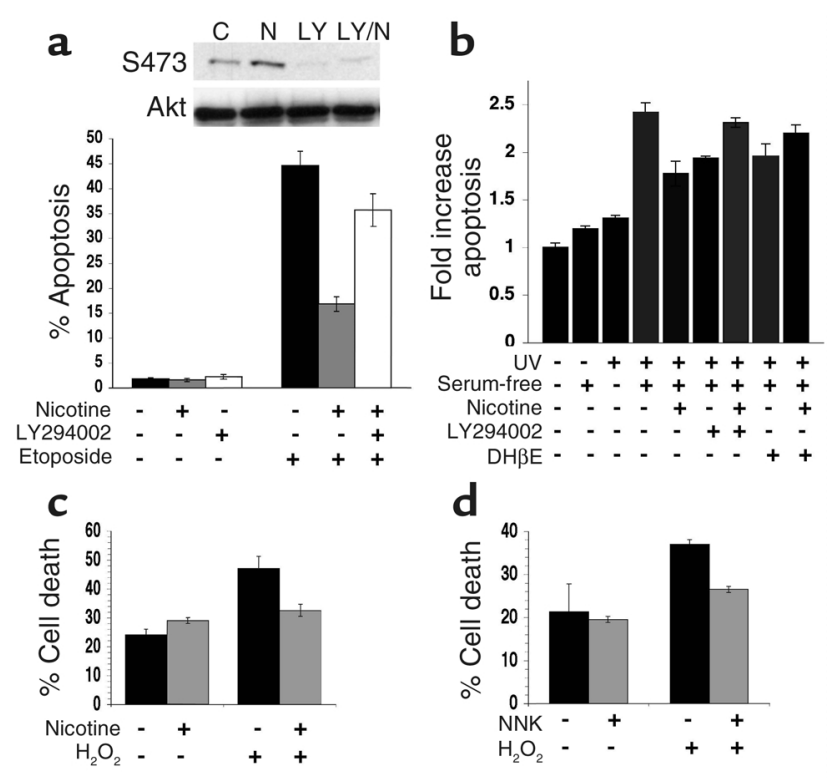

In contrast to nicotine-mediated activation of Akt, NNK-induced Akt phosphorylation was inhibited by the $\alpha_{7}$-specific antagonists $\alpha$-BTX and MLA, as well as by MCA. DH $\beta E$ was ineffective, suggesting that NNK activates Akt through $\alpha_{7}$-containing nAchRs but not through $\alpha_{3}$-or $\alpha_{4}$-containing nAchRs (Figure 3b). Further support for the role of $\alpha_{7}$ nAchRs in mediating NNK-induced Akt activation is suggested by the dose and kinetic similarities of NNK-induced Akt phosphorylation in NHBEs and SAECs, the fact that $\alpha_{7}$ is a shared functional $\alpha$ subunit between NHBEs and SAECs, and the fact that $\alpha_{7}$ antagonists inhibited NNK-induced Akt phosphorylation and kinase activity (Figure 2a, right panels) at low doses (100 nM) that are specific for $\alpha_{7}$ inhibition. Thus, nicotine and NNK likely use separate nAchRs to activate Akt.

Nicotinic activation of Akt increases epithelial cell survival. Because tobacco carcinogens promote tumor formation through damage to DNA in normal cells by form-

\section{Figure 5}

Nicotine alters NHBE phenotype. (a) Loss of contact inhibition. NHBEs were incubated with different concentrations of nicotine (filled symbols; asterisks indicate daily dosing) or complete media alone (open squares). Cell number was measured by absorbance at $540 \mathrm{~nm}$ using a 96-well microplate reader. (b) Serum starvation. NHBEs were grown in DMEM with $0.1 \%$ BSA or control media for 9 days with or without nicotine $(10 \mu \mathrm{M})$ given once on day 1 . Apoptosis was assessed using CellDeath ELISA kits. (c) Anoikis. Nicotine (left panel) or NNK (right panel) decreased anoikis, as measured using CellDeath ELISA kits. Pretreatment with LY294002 decreased protection conferred by either nicotine or NNK. Pretreatment with $\mathrm{DH} \beta \mathrm{E}$ attenuated nicotine-mediated survival (left panel), and pretreatment with $\alpha$-BTX attenuated NNK-mediated survival.

\section{Figure 4}

Nicotine-mediated Akt activation and survival of NHBEs. (a) Topoisomerase II inhibition. Nicotine $(10 \mu \mathrm{M})$ protected against etoposide-induced apoptosis, as assessed by flow cytometry. Pretreatment with LY294002 decreased nicotine-mediated survival. Parallel samples were harvested for immunoblotting (inset; C, control; N, nicotine; LY, LY294002; LY/N, LY294002 + nicotine). (b) UV irradiation. Nicotine $(10 \mu \mathrm{M})$ protected against UV irradiation-induced apoptosis, as measured using CellDeath ELISA kits. Pretreatment with LY294002 or DH $\beta E$ attenuated nicotine-mediated survival. (c and d) $\mathrm{H}_{2} \mathrm{O}_{2}$ treatment. NHBEs were pretreated with nicotine $(10 \mu \mathrm{M})(\mathbf{c})$ or NNK (d) as above, with or without $\mathrm{H}_{2} \mathrm{O}_{2}(200 \mu \mathrm{M})$. After 4 hours, cells were harvested, dead cells that exhibited cytoplasmic inclusion of $0.4 \%$ trypan blue were counted, and this number was compared with the total number of cells. At least 300 cells per sample were counted by a blinded observer.

ing DNA adducts and causing oxidative damage, we tested whether nicotinic activation of Akt would protect airway epithelial cells against modalities that cause DNA damage and induce apoptosis. When NHBEs were exposed to a topoisomerase II inhibitor, etoposide, apoptosis increased from $2 \%$ to $44 \%$, as measured by the formation of subgenomic DNA detected by flow cytometry (Figure 4a). In the presence of nicotine, etoposide-induced apoptosis was decreased by $61 \%$. LY294002 attenuated the protective effects of nicotine, and the effects of nicotine and/or LY294002 on apoptosis correlated well with changes in Akt phosphorylation detected at 24 hours (Figure 4a, inset). NNK also attenuated etoposide-induced apoptosis, but protection was only evident at time points at which Akt activation was maintained (up to 8 hours; data not shown). To test UV radiation (Figure $4 \mathrm{~b}$ ), we used an apoptosis assay that measures histone release from apoptotic cells and is more sensitive than measures of subgenomic 
DNA. UV radiation alone was a poor apoptotic stimulus, as was serum starvation alone. When serumdeprived NHBEs were exposed to UV radiation, however, apoptosis increased from 1.2- to 2.4-fold. In the presence of nicotine, UV irradiation-induced apoptosis was decreased by $58 \%$. When NHBEs were pretreated with LY294002 or DHßE, the protection conferred by nicotine was attenuated. We observed similar protective effects with administration of NNK in parallel experiments performed with $\alpha$-BTX substituted for DHßE (data not shown). When NHBEs were exposed to $\mathrm{H}_{2} \mathrm{O}_{2}$, cell death approximately doubled, as assessed by trypan blue exclusion, but nicotine (Figure 4c) or NNK (Figure 4d) inhibited the cytotoxic effects of $\mathrm{H}_{2} \mathrm{O}_{2}$. Together, these data suggest that nicotinic activation of Akt promotes the survival of NHBEs that are exposed to conditions known to cause DNA damage and stimulate apoptosis.

Nicotinic activation of Akt alters epithelial cell growth characteristics. In addition to reducing apoptosis, Akt activation can contribute to cellular transformation by increasing cell growth and decreasing dependence on exogenous growth factors or attachment to ECM. We tested whether nicotinic activation of Akt would alter these cellular parameters. When NHBEs were incubated with nicotine, either as a single dose of $10 \mu \mathrm{M}$ given on day 1 or as daily physiologic doses of $100 \mathrm{nM}$ or 1 $\mu \mathrm{M}$, differences in cell growth were apparent after approximately 7 days (Figure 5a). Untreated NHBEs became contact-inhibited, but nicotine-treated cells continued to grow until they crowded the tissue culture dishes and began to detach. These data showing dysregulated NHBE growth after nicotine administration are consistent with in vivo observations that bronchial tissues of active smokers have increased proliferative indices when compared with those of former smokers (30). With prolonged serum deprivation (Figure 5b), NHBEs underwent apoptosis that was attenuated by administration of nicotine. Likewise, nicotine (Figure 5c) or NNK (Figure 5d) inhibited death of NHBEs caused by inhibition of cellular attachment (anoikis; ref. 31). Protection conferred by nicotine was attenuated by LY294002 or by DHßE, and protection conferred by NNK was attenuated by LY294002 or by $\alpha$-BTX. These studies show that, in addition to promoting cellular survival, nicotinic activation of Akt diminishes contact inhibition and cellular dependence on exogenous growth factors or ECM.

Akt phosphorylation in NNK-treated $A / J$ mice and in buman lung cancer. To determine whether nicotinic activation of Akt was observable in vivo, we assessed lung tissue derived from the $\mathrm{A} / \mathrm{J}$ strain of mice, which is prone to develop lung tumors but which, with administration of NNK, will develop them at earlier time points with more aggressive histologies (32). We used phosphospecific antibodies against S473 in immunohistochemical experiments on formalin-fixed, paraffinembedded tissues. A/J mice treated with PBS did not exhibit phosphorylated Akt (Figure 6a). In contrast, A/J mice treated with NNK showed phosphorylated Akt in airway epithelial cells, as well as in NNK-induced tumors (Figure 6b). When protein extracts were prepared from lung tissue derived from PBS-treated or NNK-treated mice and immunoblotting was performed for phosphorylated and total Akt levels, NNKtreated mice showed a 5.5-fold increase in the ratio of phosphorylated Akt to total Akt compared with PBStreated mice (Figure 6c). These data show that exposure to NNK induces Akt activity in vivo.

We extended these studies by evaluating Akt phosphorylation in human lung cancer specimens derived from smokers. Akt phosphorylation was detected in ten of ten specimens. Staining varied from low to high levels in tumor tissues, but virtually none was detected in surrounding stroma (see representative specimen, Figure $6 \mathrm{~d}$ ). Because we were unable to obtain lung tissues from nonsmokers, we were unable to compare the levels of phosphorylated Akt in smokers versus nonsmokers. Staining with the phosphospecific S473 antibodies was completely abrogated by the inclusion of competing phosphopeptide (data not shown), indicating specificity of the phosphospecific Akt antibodies. These data are consistent with the detection of active Akt in lung tissues from NNK-treated A/J mice and show that active Akt can be detected in lung cancers derived from smokers.
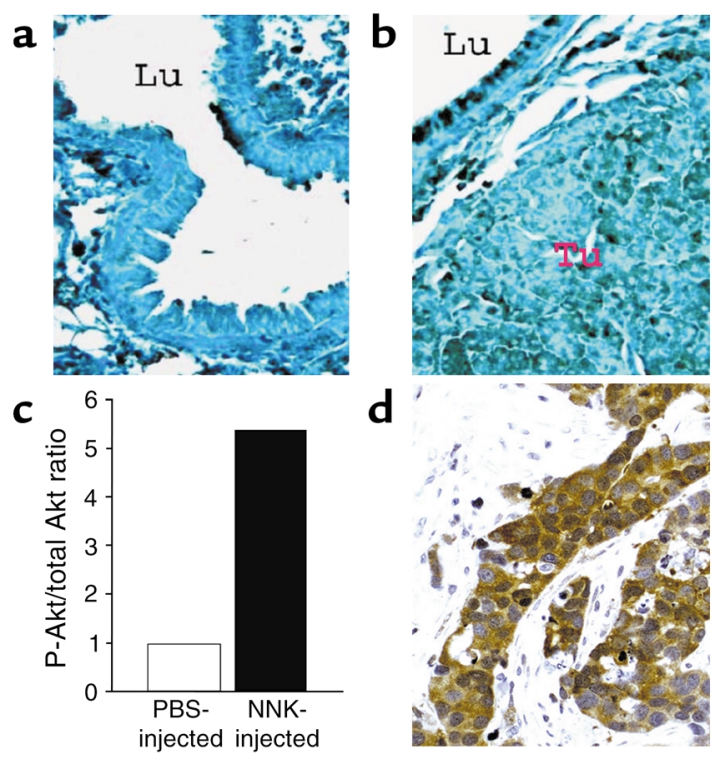

\section{Figure 6}

Detection of Akt phosphorylation in vivo. (a) Lung tissue, including epithelial lining of airway lumen ( Lu), from A/J mice given PBS orally was harvested and processed for immunohistochemistry with phosphospecific S473 antibodies as described. No staining is detectable. (b) Lung tumor (Tu) and epithelial lining of airway lumen ( $\mathrm{Lu}$ ) from A/J mice given NNK orally exhibit staining with phosphospecific S473 antibodies. (c) Quantification of phosphorylated Akt/total Akt in protein extracts derived from lung tissue from PBS- or NNK-injected mice. Quantification of immunoblots was performed using NIH Image software. (d) Phosphorylated Akt in a human lung adenocarcinoma derived from a smoker with a 48-pack-per-year smoking history. 


\section{Discussion}

Through activation of separate $\alpha$ subunit-containing nAchRs, two components of tobacco, nicotine and NNK, activate one of the best-characterized signaling pathways that promotes cellular survival, the PI3K/Akt pathway. Activation of Akt by nicotine or NNK occurred within minutes but peaked at 45-60 minutes and was maintained for hours. Although the time course of nicotine-induced Akt phosphorylation was brisk, the time course of NNK-induced Akt activation revealed an early decrease in Akt phosphorylation followed by an increase at 45-60 minutes. The mechanism of the transient decrease is unclear, but it raises the possibility that NNK-induced Akt phosphorylation might be a consequence of DNA adduct formation. Previous studies have shown that formation of DNA adducts by a purified $\alpha$ hydroxylation derivative of NNK required 2 hours of incubation with intact cells, which is longer than the time required for Akt activation in our system (33). Thus, Akt activation might precede DNA adduct formation; this would facilitate cellular survival.

The fact that two tobacco components use different nAchRs to activate the same signaling pathway raises mechanistic questions. What is the mechanism of coupling of nAchRs to the PI3K/Akt pathway? Do different nAchRs use the same mechanism to activate Akt? Although there is no precedent for activation of Akt by nAchRs containing $\alpha_{3}$ or $\alpha_{4}$, Kihara et al. recently demonstrated that, in neuronal cells, $\alpha_{7}$ nAchRs activate the PI3K/Akt pathway through activation of a member of the src family (34). Another possibility is that nicotine or NNK activates Akt through inactivation of PTEN. NHBEs and SAECs both contain wildtype PTEN (data not shown). Nicotine or NNK could inactivate PTEN by stimulating phosphorylation of residues in its C-terminus, which has been previously shown to decrease complex formation with PDZ domain-containing proteins and result in the degradation of PTEN (35). The role of src family members and PTEN inactivation in mediating nicotinic activation of Akt in airway epithelial cells is currently under investigation. Once activated by nicotine or NNK, Akt increased phosphorylation of multiple downstream components that control cellular cell cycle and protein translation, such as GSK-3, 4EBP-1, and $\mathrm{p} 70^{\mathrm{S} 6 \mathrm{~K}}$. Protein translation is likely to play an important role in Akt-mediated tumorigenesis, since an inhibitor of the ribosomal kinase $\mathrm{p} 70^{\mathrm{S} 6 \mathrm{~K}}$, CCI-779, was recently shown to inhibit $\mathrm{p} 70^{\mathrm{S} 6 \mathrm{~K}}$ activity and tumor formation in PTEN knockout mice that have high endogenous levels of Akt activity $(36,37)$.

Our studies showed that human airway epithelial cells express multiple $\alpha$ and $\beta$ nAchR subunits, but that $\mathrm{nAchR}$ subunit expression is different depending on whether the cells were derived from proximal airways (NHBEs) or distal airways (SAECs). To our knowledge, this is the most extensive analysis of nAchR subunits in non-neuronal cells, and assembly of multiple nAchR subunits into functional nAchRs explains the respon- siveness of normal human airway epithelial cells to nicotine and NNK. It is unclear whether expression of multiple nAchRs confers increased sensitivity of airway epithelial cells to tobacco components, and whether nAchR-dependent, increased sensitivity of airway epithelial cells to tobacco components might be related to tobacco-related carcinogenesis. Interestingly, the only difference in expression of nAchR subunits between normal airway epithelial cells and lung cancer cells was observed with selective expression of $\alpha_{10}$, an nAchR subunit heretofore only described in inner ear neuroepithelium $(38,39)$, in normal airway epithelial cells.

Multiple lines of evidence support our hypothesis that nicotinic activation of Akt plays a role in lung tumorigenesis. First, activation of Akt through either $\alpha_{3}-/ \alpha_{4^{-}}$ or $\alpha_{7^{-}}$containing nAchRs increased normal airway epithelial cell survival under conditions where cell death is a normal physiologic response. Inappropriate epithelial cell survival might foster increased accumulation of DNA adducts, thereby increasing mutational rates in genes such as $p 53$ and $K$-ras that appear critical for lung cancer formation. Second, in addition to enhancing survival, nicotine also partially induced a transformed phenotype in normal airway epithelial cells by decreasing contact inhibition and dependence on exogenous growth factors and adherence to ECM. Third, our ability to detect phosphorylated Akt in NNK-induced murine lung tumors as well as human lung cancers from smokers demonstrates that nicotinic activation of Akt is not limited to cultured primary cells. Interestingly, other studies have shown that nicotine can also affect lung cancer cells in vitro. Nicotine can stimulate the growth of lung cancer cells, suppress apoptosis, and increase phosphorylation of ERK (40-42).

Nicotinic alteration of other cell types may also contribute to lung tumorigenesis. In addition to increasing epithelial cell survival as described in this report, nicotine can stimulate endothelial cell growth and angiogenesis (43). This is relevant because a lung cancer precursor lesion, angiogenic squamous dysplasia (44), was recently described that recapitulates the growth-promoting effects of nicotine on epithelial and endothelial cells in vivo. Whether the angiogenic properties of nicotine require Akt activation is unclear, but multiple angiogenic factors induced by nicotine, including VEGF and angiopoietin $(45,46)$, require activation of Akt for biologic activity.

Finally, these studies might have therapeutic implications for the 169,000 Americans that will be diagnosed with lung cancer this year and the 90,000,000 Americans who are current or former smokers and are thus at permanent increased risk of developing lung cancer (47). Activation of the PI3K/Akt pathway through nAchRs could be targeted for lung cancer prevention. The fact that phosphorylated Akt was detectable in ten of ten human lung cancers derived from smokers suggests that Akt activation is maintained throughout tumorigenesis and might possibly be necessary for tumor maintenance. Although it is 
possible that Akt activation is a late event that is not causally involved in tumor progression, our in vitro data support the contention that it is an early event. Definitive proof will require analysis of lung cancer precursor lesions in smokers. If active Akt can be detected in smokers' lung cancer precursor lesions, then targeting specific nAchRs or components of the PI3K/Akt pathway might attenuate the various cellular effects of nicotine or NNK, and this could be exploited as a strategy to prevent lung cancer tumorigenesis in high-risk populations. Large clinical trials will soon open to compare the chemopreventive efficacy of two types of drugs that indirectly inhibit Akt activity, farnesyltransferase inhibitors and EGF-R antagonists, in patients at high risk for lung cancer. Changes in phosphorylation of Akt will be measured as a molecular endpoint in these trials. Interestingly, cigarette smoking causes chronic obstructive pulmonary disease (COPD), a risk factor for the development of lung cancer, more often than it causes lung cancer. If Akt activation is an early event in lung tumorigenesis, then it is possible that preventative approaches targeting the PI3K/Akt pathway might affect COPD as well.

Regardless of the timing of Akt activation in the development of lung cancer, the presence of active Akt in human lung cancers suggests that Akt might be a valid target for therapeutic approaches in patients with established NSCLC. Previously, we had demonstrated that inhibition of the PI3K/Akt pathway in NSCLC cells that had high levels of active Akt increased apoptosis (4). Greater-than-additive effects on apoptosis were observed when a small-molecule inhibitor of the PI3K/Akt pathway was combined with traditional forms of chemotherapy. A similar situation might exist in vivo. For lung cancer patients whose tumors have detectable phosphorylated Akt, drugs that inhibit the PI3K/Akt pathway might have clinical utility when used singly or in combination with traditional therapy. The fact that current therapy does not prevent most lung cancer patients from dying of their disease highlights the need for better targets and newer therapies. Our studies might also have implications for smoking cessation. Nicotine supplementation through patches, nasal sprays, chewing gum, etc., is now widely used to assist in smoking cessation. The steady-state serum concentrations of nicotine achieved with these modalities vary, but transdermal delivery of nicotine is virtually complete and can result in serum concentrations of nicotine that approximate those observed in active smokers (48). Although nicotine per se is not thought to be carcinogenic, the risks of long-term nicotine supplementation are unknown. Our data and those of Heeschen et al. (43) suggest that sustained exposure to nicotine might alter the phenotype of endothelial and/or epithelial cells in undetectable, premalignant lesions. Finally, our study focused on the roles of nAchRs and Akt activation in altering airway epithelial cell phenotype as it relates to lung cancer, which is only one of many tobacco-related cancers.
Given that $15 \%$ of all cancers worldwide are tobaccorelated, nicotinic activation of the PI3K/Akt pathway may contribute to the biology of these cancers as well.

\section{Acknowledgments}

The authors would like to thank Elise Bowman, Michael Jung, Theresa Spitzer, and Samantha Streicher for technical assistance, and K. Kellar, L. Neckers, and T. Fojo for helpful discussions and/or review of the manuscript.

1. Lawlor, M.A., and Alessi, D.R. 2001. PKB/Akt: a key mediator of cell proliferation, survival and insulin responses? J. Cell Sci. 114:2903-2910.

2. Scheid, M.P., and Woodgett, J.R. 2001. Pkb/akt: functional insights from genetic models. Nat. Rev. Mol. Cell Biol. 2:760-768.

3. Myers, M.P., et al. 1998. The lipid phosphatase activity of PTEN is critical for its tumor suppressor function. Proc. Natl. Acad. Sci. USA. 95:13513-13518.

4. Brognard, J., Clark, A.S., Ni, Y., and Dennis, P.A. 2001. Akt/protein kinase $B$ is constitutively active in non-small cell lung cancer cells and promotes cellular survival and resistance to chemotherapy and radiation. Cancer Res. 61:3986-3997.

5. Maus, A.D., et al. 1998. Human and rodent bronchial epithelial cells express functional nicotinic acetylcholine receptors. Mol. Pharmacol. 54:779-788.

6. Wang, Y., et al. 2001. Human bronchial epithelial and endothelial cells express alpha7 nicotinic acetylcholine receptors. Mol. Pharmacol. 60:1201-1209.

7. Monteggia, L.M., et al. 1995. Cloning and transient expression of genes encoding the human alpha 4 and beta 2 neuronal nicotinic acetylcholine receptor (nAChR) subunits. Gene. 155:189-193.

8. Nguyen, V.T., et al. 2000. Choline acetyltransferase, acetylcholinesterase, and nicotinic acetylcholine receptors of human gingival and esophageal epithelia. J. Dent. Res. 79:939-949.

9. Sekhon, H.S., et al. 1999. Prenatal nicotine increases pulmonary alpha7 nicotinic receptor expression and alters fetal lung development in monkeys. J. Clin. Invest. 103:637-647.

10. Bretland, A.J., Lawry, J., and Sharrard, R.M. 2001. A study of death by anoikis in cultured epithelial cells. Cell Prolif. 34:199-210.

11. Castonguay, A., Pepin, P., and Stoner, G.D. 1991. Lung tumorigenicity of NNK given orally to A/J mice: its application to chemopreventive efficacy studies. Exp. Lung Res. 17:485-499.

12. Belinsky, S.A., Devereux, T.R., Maronpot, R.R., Stoner, G.D., and Anderson, M.W. 1989. Relationship between the formation of promutagenic adducts and the activation of the K-ras protooncogene in lung tumors from A/J mice treated with nitrosamines. Cancer Res. 49:5305-5311.

13. Linnoila, R.I., et al. 1992. Peripheral airway cell marker expression in non-small cell lung carcinoma. Association with distinct clinicopathologic features. Am. J. Clin. Pathol. 97:233-243.

14. Hecht, S.S., and Hoffmann, D. 1988. Tobacco-specific nitrosamines, an important group of carcinogens in tobacco and tobacco smoke. Carcinogenesis. 9:875-884.

15. Russell, M.A., Jarvis, M., Iyer, R., and Feyerabend, C. 1980. Relation of nicotine yield of cigarettes to blood nicotine concentrations in smokers. Br. Med. J. 280:972-976.

16. Armitage, A.K., et al. 1975. Absorption and metabolism of nicotine from cigarettes. Br. Med. J. 4:313-316.

17. Lindell, G., et al. 1993. Acute effects of smoking during modified sham feeding in duodenal ulcer patients. An analysis of nicotine, acid secretion, gastrin, catecholamines, epidermal growth factor, prostaglandin E2, and bile acids. Scand. J. Gastroenterol. 28:487-494.

18. Vlahos, C.J., Matter, W.F., Hui, K.Y., and Brown, R.F. 1994. A specific inhibitor of phosphatidylinositol 3-kinase, 2-(4- morpholinyl)-8-phenyl4H-1-benzopyran-4-one (LY294002). J. Biol. Chem. 269:5241-5248.

19. Brunet, A., et al. 1999. Akt promotes cell survival by phosphorylating and inhibiting a Forkhead transcription factor. Cell. 96:857-868.

20. Cross, D., Alessi, D., Cohen, P., Andjelkovich, M., and Hemmings, B. 1995. Inhibition of glycogen synthase kinase-3 by insuylin mediated by protein kinase B. Nature. 378:785-789.

21. Burgering, B., and Coffer, P. 1995. Protein kinase B (c-akt) in phosphatidylinositol-3-OH kinase signal transduction. Nature. 376:599-602.

22. Lindstrom, J. 1997. Nicotinic acetylcholine receptors in health and disease. Mol. Neurobiol. 15:193-222.

23. Grando, S.A., et al. 1996. Activation of keratinocyte nicotinic cholinergic receptors stimulates calcium influx and enhances cell differentiation. J. Invest. Dermatol. 107:412-418.

24. Itier, V., and Bertrand, D. 2001. Neuronal nicotinic receptors: from protein structure to function. FEBS Lett. 504:118-125.

25. Gotti, C., et al. 1991. Purification and characterization of an alpha-bun- 
garotoxin receptor that forms a functional nicotinic channel. Proc. Natl. Acad. Sci. USA. 88:3258-3262.

26. Wong, E.T., et al. 1995. Pharmacological and physiological properties of a putative ganglionic nicotinic receptor, alpha 3 beta 4, expressed in transfected eucaryotic cells. Brain Res. Mol. Brain Res. 28:101-109.

27. Chavez-Noriega, L.E., et al. 2000. Characterization of the recombinant human neuronal nicotinic acetylcholine receptors alpha3beta2 and alpha4beta2 stably expressed in HEK293 cells. Neuropharmacology. 39:2543-2560.

28. Molinari, E.J., et al. 1998. Up-regulation of human alpha7 nicotinic receptors by chronic treatment with activator and antagonist ligands. Eur. J. Pharmacol. 347:131-139.

29. Tonder, J.E., and Olesen, P.H. 2001. Agonists at the alpha4beta2 nicotinic acetylcholine receptors: structure-activity relationships and molecular modeling. Curr. Med. Chem. 8:651-674.

30. Lee, J.J., et al. 2001. Long-term impact of smoking on lung epithelial proliferation in current and former smokers. J. Natl. Cancer Inst. 93:1081-1088

31. Frisch, S., and Francis, H. 1994. Disruption of epithelial cell-matrix interactions induces apoptosis. J. Cell Biol. 124:619-626.

32. Malkinson, A.M. 1998. Molecular comparison of human and mouse pulmonary adenocarcinomas. Exp. Lung Res. 24:541-555.

33. Cloutier, J.F., Drouin, R., and Castonguay, A. 1999. Treatment of human cells with $\mathrm{N}$-Nitroso (acetoxymethyl)methylamine: distribution patterns of piperidine-sensitive DNA damage at the nucleotide level of resolution are related to the sequence context. Chem. Res. Toxicol. 12:840-849.

34. Kihara, T., et al. 2001. Alpha 7 nicotinic receptor transduces signals to phosphatidylinositol 3-kinase to block A beta-amyloid-induced neurotoxicity. J. Biol. Chem. 276:13541-13546.

35. Vazquez, F., Ramaswamy, S., Nakamura, N., and Sellers, W.R. 2000. Phosphorylation of the PTEN tail regulates protein stability and function. Mol. Cell. Biol. 20:5010-5018.

36. Neshat, M.S., et al. 2001. Enhanced sensitivity of PTEN-deficient tumors to inhibition of FRAP/mTOR. Proc. Natl. Acad. Sci. USA. 98:10314-10319.

37. Podsypanina, K., et al. 2001. An inhibitor of mTOR reduces neoplasia and normalizes p70/S6 kinase activity in Pten+/- mice. Proc. Natl. Acad. Sci. USA. 98:10320-10325.

38. Lustig, L.R., Peng, H., Hiel, H., Yamamoto, T., and Fuchs, P.A. 2001. Molecular cloning and mapping of the human nicotinic acetylcholine receptor alpha10 (CHRNA10). Genomics. 73:272-283.

39. Elgoyhen, A.B., et al. 2001. Alpha10: a determinant of nicotinic cholinergic receptor function in mammalian vestibular and cochlear mechanosensory hair cells. Proc. Natl. Acad. Sci. USA. 98:3501-3506.

40. Maneckjee, R., and Minna, J.D. 1990. Opioid and nicotine receptors affect growth regulation of human lung cancer cell lines. Proc. Natl. Acad. Sci. USA. 87:3294-3298.

41. Maneckjee, R., and Minna, J.D. 1994. Opioids induce while nicotine suppresses apoptosis in human lung cancer cells. Cell Growth Differ. 5:1033-1040

42. Heusch, W.L., and Maneckjee, R. 1998. Signaling pathways involved in nicotine regulation of apoptosis of human lung cancer cells. Carcinogenesis. 19:551-556.

43. Heeschen, C., et al. 2001. Nicotine stimulates angiogenesis and promotes tumor growth and atherosclerosis. Nat. Med. 7:833-839.

44. Keith, R.L., et al. 2000. Angiogenic squamous dysplasia in bronchi of individuals at high risk for lung cancer. Clin. Cancer Res. 6:1616-1625.

45. Gerber, H.P., et al. 1998. Vascular endothelial growth factor regulates endothelial cell survival through the phosphatidylinositol 3 '-kinase/Akt signal transduction pathway. Requirement for Flk-1/KDR activation. J. Biol. Chem. 273:30336-30343.

46. Fujikawa, K., et al. 1999. Role of PI 3-kinase in angiopoietin-1-mediated migration and attachment-dependent survival of endothelial cells. Exp. Cell Res. 253:663-672.

47. Landis, S.H., Murray, T., Bolden, S., and Wingo, P.A. 1999. Cancer statistics, 1999. CA Cancer J. Clin. 49:8-31.

48. Zevin, S., Gourlay, S.G., and Benowitz, N.L. 1998. Clinical pharmacology of nicotine. Clin. Dermatol. 16:557-564. 